\title{
Vessel Or Plaque, Device Embedded In
}

National Cancer Institute

\section{Source}

National Cancer Institute. Vessel Or Plaque, Device Embedded In. NCI Thesaurus. Code C50647.

Device and/or frag ments of device are embedded in patient's vessel and/or plaque. 